\title{
BMJ Sustainability of knowledge translation open interventions in healthcare decision-making: protocol for a scoping review
}

\author{
Andrea C Tricco, ${ }^{1}$ Elise Cogo, ${ }^{1}$ Huda Ashoor, ${ }^{1}$ Laure Perrier, ${ }^{1} \mathrm{~K}$ Ann McKibbon, ${ }^{2}$ \\ Jeremy M Grimshaw, ${ }^{3,4}$ Sharon E Straus ${ }^{1,5}$
}

To cite: Tricco AC, Cogo E, Ashoor $\mathrm{H}$, et al. Sustainability of knowledge translation interventions in healthcare decision-making: protocol for a scoping review. BMJ Open 2013;3:e002970.

doi:10.1136/bmjopen-2013002970

- Prepublication history and additional material for this paper are available online. To view these files please visit the journal online (http://dx.doi.org/10.1136/ bmjopen-2013-002970).

Received 28 March 2013 Accepted 11 April 2013

This final article is available for use under the terms of the Creative Commons Attribution Non-Commercial 2.0 Licence; see http://bmjopen.bmj.com

For numbered affiliations see end of article.

Correspondence to Dr Sharon E Straus; sharon.straus@utoronto.ca
ABSTRACT

Introduction: Knowledge translation (KT also known as research utilisation, translational medicine and implementation science) is a dynamic and iterative process that includes the synthesis, dissemination, exchange and ethically sound application of knowledge to improve health. After the implementation of KT interventions, their impact on relevant outcomes should be monitored. The objectives of this scoping review are to: (1) conduct a systematic search of the literature to identify the impact on healthcare outcomes beyond 1 year, or beyond the termination of funding of the initiative of $\mathrm{KT}$ interventions targeting chronic disease management for end-users including patients, clinicians, public health officials, health services managers and policy-makers; (2) identify factors that influence sustainability of effective KT interventions; (3) identify how sustained change from KT interventions should be measured; and (4) develop a framework for assessing sustainability of KT interventions.

Methods and analysis: Comprehensive searches of relevant electronic databases (eg, MEDLINE, EMBASE, Cochrane Central Register of Controlled Trials), websites of funding agencies and websites of healthcare provider organisations will be conducted to identify relevant material. We will include experimental, quasiexperimental and observational studies providing information on the sustainability of KT interventions targeting chronic disease management in adults and focusing on end-users including patients, clinicians, public health officials, health services managers and policy-makers. Two reviewers will pilot-test the screening criteria and data abstraction form. They will then screen all citations, full articles and abstract data in duplicate independently. The results of the scoping review will be synthesised descriptively and used to develop a framework to assess the sustainability of KT interventions.

Discussion and dissemination: Our results will help inform end-users (ie, patients, clinicians, public health officials, health services managers and policy-makers) regarding the sustainability of KT interventions. Our dissemination plan includes publications, presentations, website posting and a stakeholder meeting.

\section{INTRODUCTION}

As various knowledge translation (KT also known as research utilisation, translational medicine and implementation science) interventions are found to be effective in research studies, the need to evaluate their sustainability is becoming increasingly important. In the Knowledge to Action framework proposed by Graham et al, ${ }^{1}$ sustained knowledge use is one of the main components. However, many implementation studies focus on short-term outcomes relevant to a study situation and do not address long-term outcomes in practice and policy domains. For example, in the systematic review of interventions to optimise antibiotic use, of 39 studies included, only 2 reported follow-up beyond 1 year. $^{2}$ Similarly, a systematic review of audit and feedback found that the follow-up period in studies varied from 3 weeks to 14 months and reported mixed results with respect to sustained change. ${ }^{3} \mathrm{~A}$ recent systematic review of sustainability of KT interventions identified 125 articles but few conclusions could be drawn. ${ }^{4}$ Most studies were retrospective and often lacked a definition or framework for sustainability. The authors synthesised the qualitative information to identify factors influencing sustainability.

Although several definitions of sustainability exist, we have chosen to use the definition of an intervention being in place more than 1 year after implementation or after the research or project funding period is complete. Understanding the sustainability of KT interventions requires a framework analogous to postmarketing surveillance, which is carried out by the pharmaceutical and medical device industries. As seen in the use of spironolactone following the publication of the RALES trial, ${ }^{5}$ it is important to develop an understanding of how interventions are being implemented in 'real world' settings over time and to monitor 
their effectiveness. Similar strategies need to be determined for assessing sustainability of KT interventions. Once KT interventions are implemented, their impact on relevant outcomes needs to be monitored, and the intervention itself should be monitored to determine if its 'dose' or 'formulation' needs to be adapted or replaced in the changing healthcare environment. Factors that may influence sustainability may include those related to the stakeholders, the social context of care, the organisational context and public policy. ${ }^{6}$ However, these have not been fully examined through a scoping review. As such, we propose to complete a scoping review to understand the factors that influence sustainability in healthcare decision-making and to develop a framework for assessing sustainability.

\section{METHODS AND ANALYSIS}

\section{Protocol}

A protocol for our scoping review was compiled using the rigorous methodology proposed by Arksey and $\mathrm{O}^{\prime}$ Malley, ${ }^{7}$ and others. ${ }^{8}$ The draft protocol was circulated for feedback from KT experts, systematic review methodologists and clinicians. It was modified, as necessary, and is available from the authors upon request.

\section{Eligibility criteria}

We will include experimental (randomised controlled trials (RCTs), quasi-RCTs, non-RCTs), quasi-experimental (controlled before after, interrupted time series) and observational (cohort, case control, cross-sectional) studies examining the impact of a KT intervention targeting chronic disease management in adults after more than 1 year of implementation or after the termination of research/project funding is described. Studies in all settings will be considered, including primary and specialist care; acute and long-term care; inpatient and outpatient care; and regional, provincial, national and international settings. End-users of the KT intervention will include patients, clinicians (from all disciplines), public health officials (including medical officers of health, department chairs and programme managers), healthcare managers and policy-makers (including regional, provincial/ state and federal), and will look at any clinical or healthcare system outcomes.

\section{Information sources}

We will conduct a systematic search of the published and difficult to locate or unpublished (ie, grey) literature within health. The following electronic databases will be searched from inception onwards: Medline, Embase, the Cochrane Central Register of Controlled Trials (CENTRAL) and the Cumulative Index to Nursing and Allied Health Literature (CINAHL). A search of the grey literature will be conducted using Google, and relevant discipline-based list servers, including CANMEDLIB and MEDLIB, using guidance from the Canadian Agency for Drugs and Technologies in Health. ${ }^{9}$ We will search the websites of funding agencies, and healthcare provider organisations from Canada, the USA, the UK and Australia who have similar healthcare systems and/ or similar challenges related to chronic disease management. References from included studies and relevant review articles will also be scanned to ensure literature saturation. Team members will also use their linkages with experts in the field to identify additional articles.

\section{Search strategy}

The main (MEDLINE) search strategy was developed by an experienced librarian, circulated to the team and revised, as necessary. Search terms included durability, fidelity, sustainability, institutionalisation, routinisation, longitudinal and long-term. The search was not limited by language, but was limited to adults, chronic medical diseases and humans.

Subsequently, the search strategy was peer reviewed by another experienced librarian using the Peer Review of Electronic Search Strategies (PRESS) Checklist. ${ }^{10}$ The final search strategy can be found in online supplementary appendix 1, which is available electronically on the journal's website, and was adjusted for the other databases, as necessary. The search strategies for the other databases are available from the authors on request.

\section{Study selection}

The eligibility criteria will be pilot-tested on a random sample of titles and abstracts/full-text articles. Subsequently, two investigators will independently review the retrieved abstracts to identify those that meet the inclusion criteria at the title and abstract level of screening. Assessment of potentially relevant full-text articles will be performed by two investigators independently to determine if they meet inclusion criteria.

\section{Data items and data collection process}

Two investigators will independently read each article and extract relevant data. The main focus of the studies will be categorised or "charted" ${ }^{6}$ using the following criteria:

1. What KT interventions (affecting the various stakeholder groups) targeting chronic medical disease management have been found to have sustained impact beyond 1 year on healthcare outcomes?

2. What is the effect of sustained use of a KT strategy targeting chronic medical disease management on the components of the intervention? How is it adapted over time?

Additional categories may be identified through completion of the search and in consultation with the team members. Study quality is generally not conducted during a scoping review because the aim is to identify gaps in the evidence base and to target areas for future systematic review. ${ }^{7}$

\section{Synthesis}

The data arising from our data collection process will be summarised quantitatively (using a simple numerical 
count) and qualitatively (drawing on the descriptive analytical method) using thematic analysis with the N-Vivo software (V.10). Through this process, we will be able to identify where gaps exist in the literature, as well as the research area(s) which require a systematic review or primary research. We will use our results to (1) determine factors that influence sustainability of KT interventions, (2) determine how sustainability of KT interventions should be measured and (3) develop a framework for assessing the sustainability of KT interventions.

\section{DISCUSSION AND DISSEMINATION}

The results of the proposed scoping review will be used to develop a framework for assessing sustainability for chronic disease management of KT interventions. This framework will advance both the science and practice of KT. Furthermore, it will be of utility to researchers, funders and those interested in implementing KT interventions including clinicians, healthcare managers and policy-makers, among others. To our knowledge, there has been no previous attempt to understand the impact of sustained KT interventions or the factors that influence sustainability.

We will ensure broad dissemination of our research findings through multiple KT strategies. These include publications in open-access, peer-reviewed journals, conference presentations, posting our results on our KT Canada website (http://ktclearinghouse.ca/ktcanada) and creating user-friendly executive summaries of our results. We will also hold a dissemination meeting with our key stakeholders, including researchers, funders, clinicians, healthcare managers and policy-makers, among others.

\section{Author affiliations}

${ }^{1}$ Li Ka Shing Knowledge Institute, St Michael's Hospital, Toronto, Ontario, Canada

${ }^{2}$ Department of Clinical Epidemiology \& Biostatistics, McMaster University, Hamilton, Ontario, Canada

${ }^{3}$ Clinical Epidemiology Program, Ottawa Hospital Research Institute, Ottawa, Ontario, Canada

${ }^{4}$ Department of Medicine, Faculty of Medicine, University of Ottawa, Ottawa, Ontario, Canada

${ }^{5}$ Department of Medicine, University of Toronto, Toronto, Ontario, Canada
Acknowledgements This scoping review was funded by the Canadian Institutes of Health Research through Knowledge Translation Canada. ACT is funded by a Canadian Institutes of Health Research/Drug Safety and Effectiveness Network (CIHR/DSEN) New Investigator Award in Knowledge Synthesis. JMG is funded by a Tier 1 Canada Research Chair in Health Knowledge Transfer and Uptake. SES is funded by a Tier 1 Canada Research Chair in Knowledge Translation. We thank Becky Skidmore for peer reviewing the literature search.

Contributors ACT helped conceive the study design and also draft the protocol. EC and HA helped draft the protocol. KAM and JMG helped conceive the study and obtain funding for it, as well as edited the draft protocol. LP developed and executed the search strategy and edited the draft protocol. SES conceived the study, obtained funding for it, and wrote the protocol. All authors read and approved the final manuscript.

Funding This work was supported by the Canadian Institutes of Health Research grant number CRI 88368.

Competing interests None.

Provenance and peer review Not commissioned; internally peer reviewed.

Data sharing statement Unpublished study data, such as the search strategies for the other databases, are available upon request to the corresponding author.

\section{REFERENCES}

1. Graham I, Logan J, Harrison M, et al. Lost in knowledge translation: time for a map? J Contin Educ Health Prof 2006;26:13-24.

2. Arnold SR, Straus SE. Interventions to improve antibiotic prescribing practices in ambulatory care. Cochrane Database Syst Rev 2005;2: CD003539.

3. Ivers N, Jamtvedt G, Flottorp S, et al. Audit and feedback: effects on professional practice and healthcare outcomes. Cochrane Database Syst Rev 2012;4:CD000259.

4. Stirman S Wiltsey, Kimberly J, Cook N, et al. The sustainability of new programs and innovations: a review of the empirical literature and recommendations for future research. Implement Sci 2012;7:17.

5. Juurlink DN, Mamdani MM, Lee DS, et al. Rates of hyperkalemia after publication of the Randomised Aldactone Evaluation Study. N Engl J Med 2004;351:543-51.

6. Grol R, Grimshaw J. Evidence-based implementation of evidence-based medicine. Jt Comm J Qual Improv 1999;25:503-13.

7. Arksey $\mathrm{H}$, O'Malley L. Scoping studies: towards a methodological framework. Int J Soc Res Methodol 2005;8:19-31.

8. Levac D, Colquhoun H, O'Brien KK. Scoping studies: advancing the methodology. Implement Sci 2010;5:69.

9. Canadian Agency for Drugs and Technologies in Health. Grey Matters: a practical search tool for evidence-based medicine [Electronic source]. April 2008 (updated January 2013). http://www. cadth.ca/resources/grey-matters (last accessed Mar 2013).

10. Sampson M, McGowan J, Cogo E, et al. An evidence-based practice guideline for the peer review of electronic search strategies. $J$ Clin Epidemiol 2009;62:944-52. 